Check for updates

Cite this: Nanoscale Adv., 2019, 1, 1117

\title{
Negative Poisson's ratio in 2D life-boat structured crystals $\uparrow$
}

\author{
Ruhao Fang, (D) ad Xiangyuan Cui, ${ }^{* b c}$ Catherine Stampfl, ${ }^{\text {a }}$ Simon P. Ringer ${ }^{\text {bc }}$ \\ and Rongkun Zheng (D) *acd
}

Two-dimensional auxetic materials with negative Poisson's ratio expand in response to a tensile strain in cross-section. Such counter-intuitive behaviours have been mainly ascribed to concave structures with ideal rigid ball-stick models. Here, based on first-principles calculations, we systematically analyze the mechanical behaviours of three life-boat structured two-dimensional (2D) materials and report two new auxetic materials: $\delta$-arsenic and $\delta$-graphane. The calculated Poisson's ratio values are correlated with Young's modulus, cohesive energy, and valence shell electron pair repulsion of isostructures. Combining with previous research, we provide a self-consistent explanation of the origin of the $2 \mathrm{D}$ in-plane negative Poisson's ratio and an algorithmic route to discover new auxetic materials by comparing the energy restored in bond rotation and stretch.

Received 16th November 2018

Accepted 11th December 2018

DOI: $10.1039 /$ c8na00352a

rsc.li/nanoscale-advances

unit cell) tends to behave in a positive feedback to strain,

\section{Introduction}

The Poisson's ratio (PR) of a material is defined as $\nu_{j i}=-\varepsilon_{j} / \varepsilon_{i}$, where $\varepsilon_{i}$ is an applied strain in the active (i-axis) direction and $\varepsilon_{j}$ is the corresponding strain in the transverse $(j$-axis) direction. It reflects the response in the transverse direction to a uniaxial strain in the longitudinal direction. For normal solids, Poisson's ratio usually falls in the range $0-0.5,{ }^{1}$ which behaves as a geometrically inverse feedback to external strain. However, counter-intuitively, there exist a group of materials with negative Poisson's ratio (NPR), namely auxetic materials, exhibiting expansion in the transverse direction when a tensile strain is applied in the longitudinal direction. ${ }^{2}$ Materials with NPR are of great interest in biomedicine, ${ }^{3}$ fasteners, ${ }^{4}$ tougher composites, ${ }^{5}$ tissue engineering applications ${ }^{6}$ for their enhanced toughness ${ }^{7}$ and shear resistance. ${ }^{8}$ R. Lakes firstly correlated these phenomena to the unique mechanical structure of the auxetic materials. ${ }^{9}$ Since ordinary crystals commonly have the convex structure (bonds in which are located at the edge of the unit cell), the perpendicular cross section of the unit cell tends to decrease under a tensile strain, compelling the atoms to shrink in the transverse direction. On the contrary, concave structure (the bonds in which are located inside or on the surface of the

${ }^{a}$ School of Physics, The University of Sydney, New South Wales, 2006, Australia. E-mail: rongkun.zheng@sydney.edu.au

${ }^{b}$ School of Aerospace, Mechanical and Mechatronic Enigineering, The University of Sydney, Sydney, New South Wales, 2006, Australia.E-mail: carl.cui@sydney.edu.au ${ }^{c}$ Australian Centre for Microscopy and Microanalysis, The University of Sydney, Sydney, New South Wales, 2006, Australia

${ }^{d}$ Nano Institute, The University of Sydney, Sydney, New South Wales, 2006, Australia $\dagger$ Electronic supplementary information (ESI) available. See DOI: $10.1039 / \mathrm{c} 8 \mathrm{na00352a}$ exhibiting an NPR. ${ }^{10}$ For three-dimensional (3D) auxetic materials, such as $\alpha$-cristobalite, ${ }^{11}$ the A7 structure, ${ }^{12}$ the cubic metals, ${ }^{13}$ and the honeycomb,${ }^{14}$ the more concave the structure is, the higher the NPR will be. ${ }^{15}$ In such an ideal treatment, crystal structures were assumed to be made of rigid sticks linked by flexible hinges (i.e. ball-stick model), neglecting the chemical effects arising from distinct electronic configurations at different bond angles.

Since the discovery of graphene in 2004, two-dimensional (2D) materials have been under intensive research for diverse applications due to their larger elastic strain duration than their bulk counterparts ${ }^{16}$ In particular, $\alpha$-phosphorene was the first auxetic 2D material discovered in 2014, with an NPR of -0.027 in the zigzag direction. ${ }^{17}$ Its peculiar geometry in the unit cell is formed by three P-P bonds and a lone pair, each with two $\mathrm{sp}^{3}$ orbital electrons from neighbouring phosphorus atoms. ${ }^{18}$ Crystals with such bonding framework may result in four tetragonal systems ( $\alpha-, \beta-, \gamma-$, and $\delta$-phase) and more monoclinic allotropes (such as $\varepsilon$-phase), as found in phosphorene, ${ }^{18,19}$ and arsenic. ${ }^{20}$ Similarly, graphane, a hydrocarbon with the $\mathrm{C}: \mathrm{H}$ ratio $=1: 1$, may also form in these structures (see ref. 21 and references therein $)^{21}$ since the electrons around carbon atoms have similar distribution: three pairs of $\mathrm{C}-\mathrm{C} \mathrm{sp}^{3}$ bonding electrons and one pair of $\mathrm{C}-\mathrm{H} \mathrm{sp}{ }^{3}$ bonding electrons taking the place of the lone pair in phosphorene or arsenic. Subsequently, many $2 \mathrm{D}$ materials with puckered structure, such as SnSe, ${ }^{22}$ penta-graphene ${ }^{23}$ and $\mathrm{W}_{2} \mathrm{C}$, $^{24}$ were reported to have NPR. Recently, $\delta$-phosphorene, one of the 2D tetragonal-system phosphorene allotropes with the so-called life-boat structure, has been reported with a significantly low NPR $\sim-0.267 .{ }^{25}$ Moreover, 1T-phased 2D transition metal dichalcogenides 
(TMDCs) have been systematically investigated and some possess positive PR (PPR) while others have NPR. ${ }^{26}$ The variation of PR values was attributed to the distinct $\mathrm{p}-\mathrm{d}$ orbital electronic interactions between the chalcogen $\mathrm{p}$ orbitals and the transition-metal d orbitals. This highlights the fundamental role of chemical bonding in affecting the PR values for isostructural materials.

Although different elements with isostructures are expected to have different PR values, we notice that the underlying mechanism of NPR from a chemical $\mathrm{sp}^{3}$ bonding perspective is unclear. According to Lakes' theory, any material with concave life-boat structure (see Fig. 1) should have an NPR. In a practical process, tensile strain enlarges the length of the bonds, and the valence shell electron pair repulsion (VSEPR) tends to limit the bonds rotational freedom. As such, the NPR values of isostructural systems consisting of different elements should be related to their corresponding elemental electronic configurations. To study the intrinsic logic behind PR values of isostructural systems, we select the life-boat structure (one of the 2D structure with reported lowest PR) and calculate the PR values for $\delta$-arsenic, ${ }^{20} \delta$-phosphorene and $\delta$-graphane ${ }^{21}$ (a mechanically stable hydrocarbon with the same structure as $\delta$ phosphorene) and correlate NPR values with their chemical structures. Moreover, combining with previous auxetic material research, we analyse and summarize the correlation between
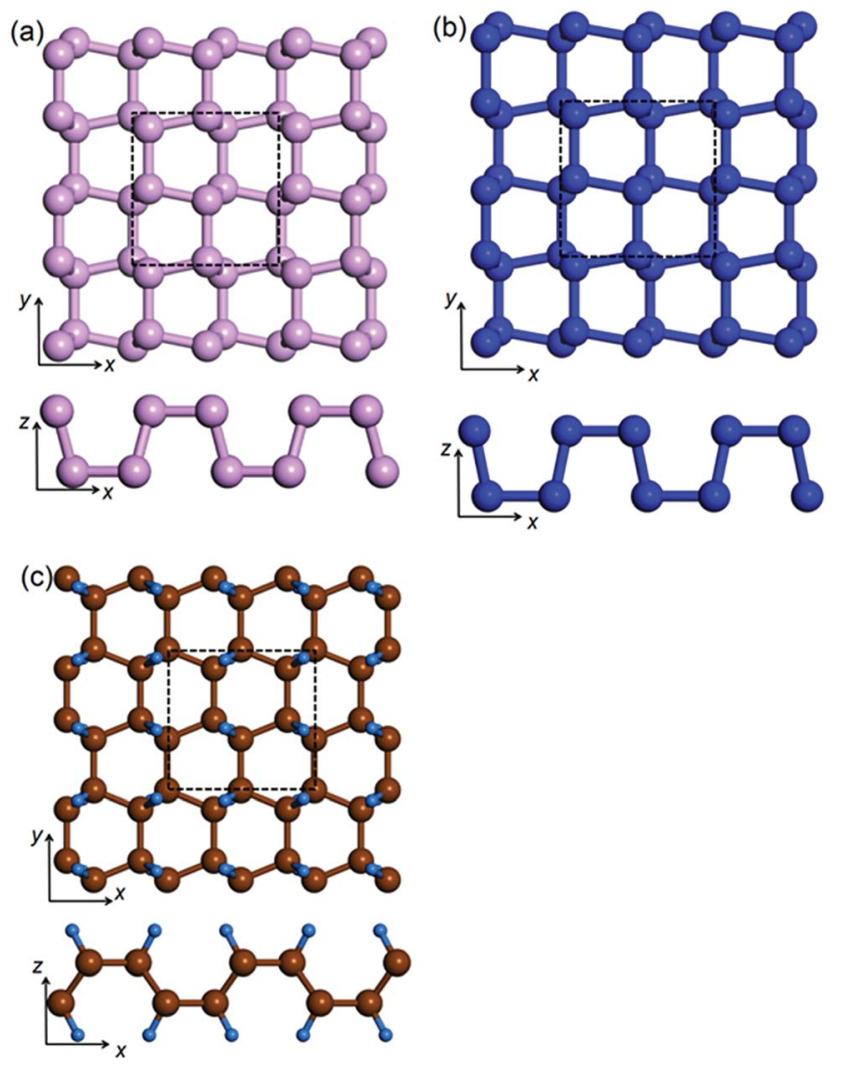

Fig. 1 The atomic structures of $\delta$-phosphorene (a), $\delta$-arsenic (b) and $\delta$-graphane (c), which all have the life-boat shape in the $x$-direction. The unit cell of each material is marked in the dash-line square. $x-, y$ and $z$-direction are defined in the diagram respectively. the NPR and electronic configurations and provide an operating route to find new $2 \mathrm{D}$ auxetic materials.

\section{Methods}

\section{DFT computational details}

The first-principles calculations were based on the density functional theory (DFT) as implemented in the Vienna ab initio simulation package (VASP) and were used to determine the atomic structure and electronic properties of atomic-layer $\delta$ phosphorene, $\delta$-arsenic and $\delta$-graphane under certain strains. We employed the generalized gradient approximation (GGA) of Perdew-Burke-Ernzerhof $(\mathrm{PBE})^{27}$ with van der Waals (VdW) corrections in exchange and correlation functions. ${ }^{28}$ Convergence testing was performed for cut-off energies from $100 \mathrm{eV}$ to $1000 \mathrm{eV}$ and $k$-point meshes from $4 \times 4 \times 4$ to $24 \times 24 \times 24$. In a balance of time consumption and accuracy, a cut-off energy of $400 \mathrm{eV}$ for phosphorene and graphene and $800 \mathrm{eV}$ for arsenic were adopted to ensure total energy convergence. A sampling of the reciprocal space Brillouin zone was set as a grid of $8 \times 8 \times 8$ $k$-points. To simulate $2 \mathrm{D}$ crystals, a unit cell with periodic boundary conditions was used, in which a vacuum space of at least $20 \AA$ was applied to minimize the interaction from $z$ direction neighbours.

The uniaxial strain is defined as

$$
\varepsilon_{i}=\frac{\Delta a_{i}}{a_{i 0}}
$$

where $i$ represents a certain direction such as $x$ or $y, \Delta a_{i}$ represents the difference between the lattice constant $a_{i}$ under a certain strain and $a_{i 0}$ is that of the strain-free system. For each uniaxial strain applied, the lattice constants in the transverse direction were optimized. The Poisson's ratio is obtained via the fitting of $\varepsilon_{j}=-\nu_{j i} \varepsilon_{i}+\nu_{2} \varepsilon_{i}^{2}+\nu_{3} \varepsilon_{i}^{3}$ within $5.5 \%$ tensile strain in each case.

The stress is defined as

$$
\sigma_{i}=\frac{1}{V} \frac{\mathrm{d} U}{\mathrm{~d} \varepsilon_{i}}
$$

where $V$ is the volume of the unit cell, which is the product of the two in-plane lattice constants and the effective thickness (defined as the vertical difference between the highest and the lowest atom plus atomic radius), $U$ is the total energy of the system. Young's modulus can be obtained from the slope of the stress-strain curve in the elastic region.

\section{Results and discussion}

\section{Crystal structure}

The unit cell of monolayer $\delta$-phosphorene $(\delta$-arsenic and $\delta$ graphane) consisting of 8 phosphorus (arsenic and carbonhydrogen) atoms, forming two atomic layers, are shown in Fig. 1. The lattice constants are shown in Table 1. The lattice constants in our calculations are systematically smaller than previously reported values (in the bracket in Table 1) due to the inclusion of VdW correction in our work. We found that VdW interaction among neighbouring, but not chemically bonded, 
Table 1 The calculated atomic structures and cohesive energy of 2D $\delta$-phosphorene, $\delta$-arsenic and $\delta$-graphane, compared with other values in the literature (brackets)

$\begin{array}{ll}\text { Crystal } & \delta \text {-Phosphorene } \\ \text { Lattice constant } a(\AA) & 5.50(5.56 \text { (ref. 18)) } \\ \text { Lattice constant } b(\AA) & 5.40(5.42 \text { (ref. 18)) } \\ \text { Cohesive energy } E_{\mathrm{c}}(\mathrm{eV} \text { per atom) } & 3.67(3.23 \text { (ref. 18)) } \\ \text { Atom radius }(\mathrm{pm}) & 195 \\ \text { Bond length }(\AA) & 2.27\end{array}$

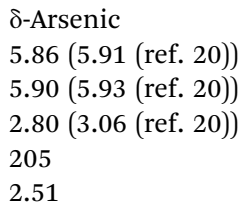

$\delta$-Graphane

$3.76(3.83$ (ref. 21))

3.69 (3.83 (ref. 21))

$3.29^{a}$

$170^{a}$

$1.09^{a}$

${ }^{a}$ Values for $\delta$-graphane are for carbon and C-C bonds.

atoms leads to non-negligible changes in the lattice constants even in these monolayer $2 \mathrm{D}$ materials. The life-boat shape of these structures along the $x$-direction and the zigzag shapes in the $y$-direction results in anisotropic mechanical responses in different directions, including anisotropic Poisson's ratios. We also tested solid nitrogen and antimony in this structure and confirmed these structures were mechanically unstable.

\section{Poisson's ratios and mechanical properties}

Table 2 shows the calculated Poisson's ratio $\left(\nu_{x y}\right.$ and $\left.\nu_{y x}\right)$ and mechanical properties for $\delta$-phosphorene, $\delta$-arsenic and $\delta$-graphane subjected to an up-to $5.5 \%$ tensile strain in either $x$ or $y$-direction. The negative PRs are found in both directions for all these life-boat structured crystals. This indicates that the occurrence of NPR can be expected based on a purely geometric consideration. However, the PR values vary from -0.072 to -0.148 in the $x$-direction and -0.090 to -0.265 in the $y$-direction for these similar puckered structures of different elements. Evidently, the NPR value is strongly affected by the chemical structure.

Table 3 shows the comparison of NPR values of recently predicted $2 \mathrm{D}$ materials, where $\delta$-graphane ranks $6^{\text {th }}$.

\section{Mechanical behaviour under tensile strain}

To understand the strain behaviours of the auxetic materials, we systematically calculated the volume, total energy and stress as a function of tensile strain (up to $5.5 \%$ ) of $\delta$-phosphorene, $\delta$ graphane and $\delta$-arsenic (shown in Fig. $2(\mathrm{a}-\mathrm{i})$ ). During the calculation for volume-strain curves, the volume $(V)$ of the 2D unit cell is defined as the product of the two in-plane lattice constants and the effective layer thickness. ${ }^{29}$ For positive Poisson's ratio (PPR) materials, enlarged longitudinal (the direction of applied strain) lattice constant reduces the other two in the transverse and vertical directions. The volume of NPR materials with the life-boat structure increases linearly-like upon tensile

Table 2 The calculated Poisson's ratio values and mechanical properties of different life-boat structured crystals

\begin{tabular}{llll}
\hline Property & $\delta$-Phosphorene & $\delta$-Arsenic & $\delta$-Graphane \\
\hline Poisson's ratio $\nu_{x y}$ & -0.148 & -0.072 & -0.080 \\
Poisson's ratio $\nu_{y x}$ & -0.265 & -0.090 & -0.160 \\
Young's modulus $E_{x}(\mathrm{GPa})$ & 83.1 & 67.1 & 200 \\
Young's modulus $E_{y}(\mathrm{GPa})$ & 145.2 & 97.3 & 340
\end{tabular}

strain, but faster than PPR materials since both in-plane lattice constants increase upon longitudinal tensile strain. The ratio of the increment of volume to the strain is approximately 1 for all the three $2 \mathrm{D}$ materials studied here, while the ratio for traditional cubic mononitrides is less than 0.6. ${ }^{30}$ The parabolic trend of total energy-strain curves is similar to those of non-auxetic behaviour materials, which indicates that for both PPR and NPR materials, the external work done upon elastic strain is transformed into strain energy due to deformation.

The anisotropic mechanical responses in different directions are evident in the calculated strain-stress curves, where the stress increases monotonously, but slows down as the tensile strain rises, which implies the PR is a strain-dependent property. These facts above indicate that the NPR phenomenon is more counter-intuitive in macroscopic geometrical behaviour than in mechanical behaviours.

\section{Bond rigidity}

The different PR values of TMDCs was ascribed to the $t_{2 g^{-}}$ bonding orbitals with different d-orbital electrons ${ }^{26}$ yet neither phosphorene nor graphane has d-orbital electrons. Here we put forward a more general explanation. For a given auxetic structure, the length of rigid bonds in an ideal ball-stick model is unchanged and the sticks can rotate freely around the "knot" (ball) under applied strain. However, if the length of the bonds is changeable, the "sticks" tend to extend to bear the strain in order to reduce the extent of change in the transverse lattice

Table 3 Comparison of the negative Poisson's ratios in recently predicted 2D materials

\begin{tabular}{|c|c|c|c|}
\hline System & Method & $\begin{array}{l}\text { Poisson's } \\
\text { ratio } \nu_{x y}\end{array}$ & $\begin{array}{l}\text { Poisson's } \\
\text { ratio } \nu_{y x}\end{array}$ \\
\hline$\alpha$-Phosphorene (BP) & $\mathrm{PBE}^{17}$ & 0.046 & -0.027 \\
\hline$\delta$-Phosphorene & $\begin{array}{l}\text { PBE + D2 } \\
\text { (ref. 25) }\end{array}$ & $\begin{array}{l}-0.148 \\
(-0.158)^{a}\end{array}$ & $\begin{array}{l}-0.265 \\
(-0.267)^{c}\end{array}$ \\
\hline$\delta$-Arsenic & $\mathrm{PBE}+\mathrm{D} 2$ & -0.072 & -0.090 \\
\hline$\delta$-Graphane & $\mathrm{PBE}+\mathrm{D} 2$ & -0.080 & -0.160 \\
\hline Penta-graphene & $\mathrm{PBE}^{23}$ & -0.068 & - \\
\hline SnSe & $\mathrm{PBE}^{22}$ & - & -0.17 \\
\hline $1 \mathrm{~T}-\mathrm{MoS}_{2}$ & $\mathrm{PBE}^{26}$ & -0.07 & -0.07 \\
\hline $1 \mathrm{~T}-\mathrm{WSe}_{2}$ & $\mathrm{PBE}^{26}$ & -0.2 & -0.2 \\
\hline $1 \mathrm{~T}-\mathrm{TcTe}_{2}$ & $\mathrm{PBE}^{26}$ & -0.38 & -0.38 \\
\hline $\mathrm{W}_{2} \mathrm{C}$ & $\mathrm{PBE}^{24}$ & -0.33 & -0.36 \\
\hline
\end{tabular}



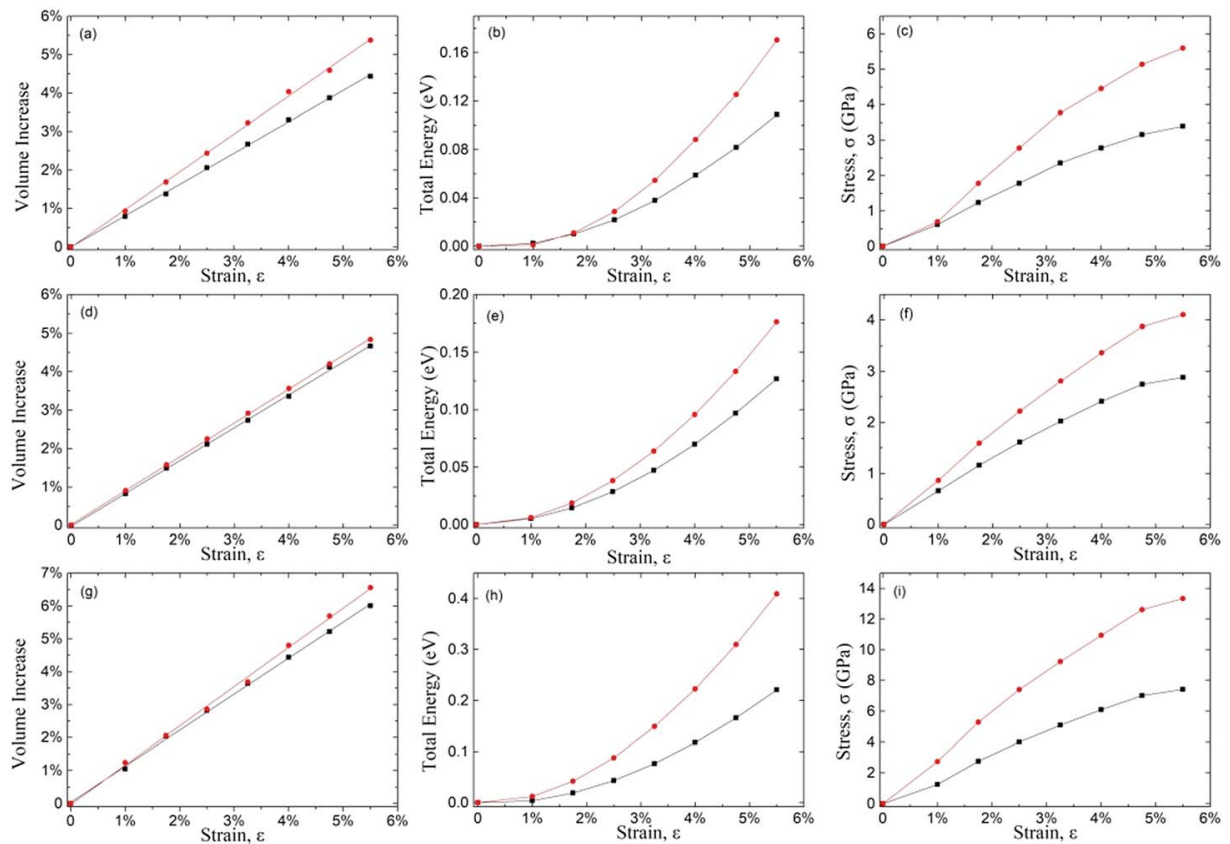

Fig. 2 The volume-strain curve, the total energy-strain curve of and the stress-strain curve of $\delta$-phosphorene $(a-c), \delta$-arsenic $(d-f)$ and $\delta$ graphane $(\mathrm{g}-\mathrm{i})$, in which the black curve represents the behaviour in the $x$-direction and the red curve in the $y$-direction.

constant. To quantify the rigidity of the chemical bonds among these three systems, we compare the cohesive energies of these three systems. From $\delta$-arsenic, $\delta$-graphane to $\delta$-phosphorene, the increasing high cohesive energy values reflect that it is more difficult to elongate the chemical bond (Table 1). For those structures with greater stiffness, they tend to rearrange the geometrical structure so as to extend the transverse lattice constant in responding the longitudinal tensile strain, which means smaller PR; hence $\nu_{\text {phosphorene }}<\nu_{\text {graphane }}<\nu_{\text {arsenic }}$.

For a given structure, the stiffness partially reflects the rigidity of the bond. Thus, we also calculated the effective Young's modulus for each system based on the stress-strain curves (shown in Table 2). $\delta$-Phosphorene and $\delta$-arsenic have the greatest similarity as they are both isostructures with similar charge distribution. The phosphorus-based life-boat structure has a greater Young's modulus $(83.1 \mathrm{GPa}$ in the $x$-direction and 145.2 $\mathrm{GPa}$ in the $y$-direction) than the arsenic-based one (67.1 GPa in the $x$-direction and 97.3 GPa in the $y$-direction), which implies $\delta$-phosphorene has stronger bonds than $\delta$ arsenic. Also, $\delta$-phosphorene has smaller values of PR than $\delta$ arsenic, which is consistent with our previous discussion.

Though $\delta$-graphane has the highest Young's modulus (200 GPa in the $x$-direction and $340 \mathrm{GPa}$ in the $y$-direction), it is $\delta$-phosphorene that shows the most obvious auxetic behaviour, with the lowest PR, -0.265 in the $y$-direction and -0.148 in the $x$-direction (in agreement with the previous work ${ }^{25}$ ). This indicates that the stiffness is a collective macroscopic property, which reflects how difficulty we change the length of the whole material rather than just a specific chemical bond. This paradox is also observed in 1T-TMDCs. ${ }^{26}$ The variation of NPR values for isostructures is believed to be determined by their distinct elemental electronic configurations. To fully explain this phenomenon, we calculate the electron charge distribution (shown in Fig. 3) of the three systems, which reveals a deeper understanding.

\section{$\mathrm{sp}^{3}$ VSEPR}

Ideal geometrical analysis of auxetic materials assumed that bonds can freely rotate around connected atoms. However, the valence shell electron pair repulsion (VSEPR) ${ }^{31}$ among neighbouring bonding or lone paired electrons constrain the rotation. In a life-boat structure, there are four $\mathrm{sp}^{3}$ orbitals around each $\mathrm{P}$ (As, C) atom, three of which are occupied by pairs of bonding electrons from neighbouring $\mathrm{P}$ (As, C) atoms, and the last one is occupied by 2 lone paired electrons $(\delta$-phosphorene and $\delta$-arsenic) or 2 bonding electrons from the $\mathrm{C}-\mathrm{H}$ bond $(\delta$ graphane). Fig. 3 shows the charge distribution of these three systems, from which the lone pairs in $\delta$-phosphorene and $\delta$ arsenic are closer to $\mathrm{P}$ or As atoms compared to the $\mathrm{C}-\mathrm{H}$ orbital electrons in $\delta$-graphane to the $\mathrm{C}$ atoms. When a tensile strain is applied, P-P and As-As bonds are easier to rotate towards the paired electrons as the paired electrons can move further away from the $\mathrm{P}$ or As atoms. On the other hand, $\mathrm{C}-\mathrm{H}$ bonds show greater VSEPR to $\mathrm{C}-\mathrm{C}$ bonds than that among lone pair to $\mathrm{P}-\mathrm{P}$ or As-As bond. Smaller VSEPR allows $\mathrm{P}-\mathrm{P}$ bonds in $\delta$-phosphorene to rotate more freely than $\mathrm{C}-\mathrm{C}$ bonds in $\delta$-graphane. As a result, $\nu_{\text {phosphorene }}$ is greater than $\nu_{\text {graphane, }}$ and $\nu_{\text {graphane }}$ is closer in value to $\nu_{\text {arsenic }}$ than $\nu_{\text {phosphorene }}$.

\section{Deformation mechanism}

To systematically understand the microscopic auxetic behaviour of the life-boat structured materials, we plot a diagram of the deformation mechanism in a unit cell under longitudinal 
(a)
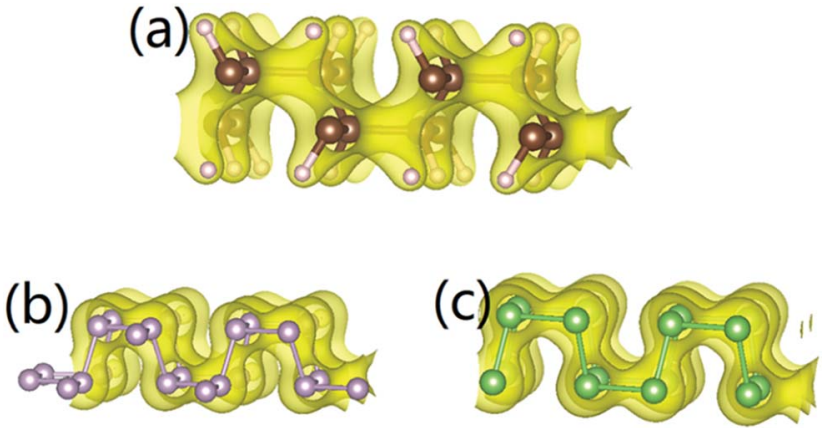

Fig. 3 The charge distribution of $\delta$-graphane (a), $\delta$-phosphorene (b) and $\delta$-arsenic (c) in which the isosurfaces are 0.1 electron per $\AA^{3}$ for $\delta$ phosphorene and $\delta$-arsenic, and 0.32 electron per $\AA^{3}$ for $\delta$-graphane, respectively. Each phosphorus or arsenic atom has 5 outer electrons, 3 of them share $3 \mathrm{P}-\mathrm{P} \mathrm{sp}^{3}$ or As-As $\mathrm{sp}^{3}$ orbitals with electrons from neighboured atoms and the rest lone-paired two electrons occupy the last $\mathrm{sp}^{3}$. Each carbon has 4 outer electrons, 3 of them share $3 \mathrm{C}-\mathrm{C} \mathrm{sp}^{3}$ and the rest share a $\mathrm{C}-\mathrm{H} \mathrm{sp}{ }^{3}$ with one electron from hydrogen. The lone pairs in $\delta$-phosphorene and $\delta$-arsenic are more closed to $P$ and $A s$ atoms, allowing $\mathrm{P}-\mathrm{P}$ and $\mathrm{As}-\mathrm{As}$ bonds to rotate more freely.

tensile strain in the $y$-direction (Fig. 4). The ball-stick model in the figure is used to represent either the carbon framework in $\delta$ graphane or the unit cell of $\delta$-phosphorene or $\delta$-arsenic. There are 3 different motions including 2 translational motions for both the $x$ - and $y$-directions (marked red and black, respectively) and 1 rotational motion (marked in blue) during the rearrangement of the 8 atoms (or 8 pairs of $\mathrm{CH}$ for $\delta$-graphane) in the unit cell. When a tensile strain is applied, the outer four atoms $\left(\mathrm{A}_{1}, \mathrm{~B}_{1}, \mathrm{C}_{2}\right.$ and $\mathrm{D}_{2}$ ) in the $y$-direction move outwards correspondingly (as shown by black arrows). The two bonds $\mathrm{D}_{1} \mathrm{D}_{2}$ and $\mathrm{A}_{2} \mathrm{D}_{2}$ constrain the movement of atom $D_{2}$ and pull the $D_{1}$ and $A_{2}$ (so as to $A_{1} B_{1}$ and $C_{2}$ due to the symmetry as shown in Fig. 4). The bonds $A_{2} B_{2}$ and $\mathrm{C}_{1} \mathrm{D}_{1}$ are slightly stretched and store the energy from the work done by tensile strain and the bonds $\mathrm{A}_{1} \mathrm{~A}_{2}, \mathrm{~B}_{1} \mathrm{~B}_{2}, \mathrm{C}_{1} \mathrm{C}_{2}$ and $\mathrm{D}_{1} \mathrm{D}_{2}$ are forced to rotate (as shown by blue arrows). However, the repulsion from the paired electrons in the last $\mathrm{sp}^{3}$ orbital (either in the $\mathrm{C}-\mathrm{H}$ bond or a suspended orbital) around all 8 atoms limits the rotation. Under this mechanism, the energy stored in the stretched $\mathrm{A}_{2} \mathrm{~B}_{2}$ and $\mathrm{C}_{1} \mathrm{D}_{1}$ bonds should balance that in the compressional deformation of electron density by bond rotation. As a result, the rotations reduce the effective thickness and push the inner 4 atoms $\left(\mathrm{A}_{2}, \mathrm{~B}_{2}, \mathrm{C}_{1}\right.$ and $\left.\mathrm{D}_{1}\right)$ outwards as well (as shown by the red arrows). The collection of these processes manifests the enlarged transverse lattice constant.

\section{Pattern to find new auxetic material}

Combining our analysis and previous NPR research, we can now summarize the origin of the NPR phenomenon for 2D materials at the atomic level. Here we define $a$ (x-direction) and $b(y$ direction) as the two in-plane lattice constants of a 2D material unit cell, and $c$ as the reduced effective thickness in the $z$ direction. For a 2D material, the effective thickness is much less than $a$ and $b$, and the decrease of $c$ is predominantly caused by the flatting of the chemical bonds connecting interlayer atoms under a tensile strain. As a result, the effective volume enlarges
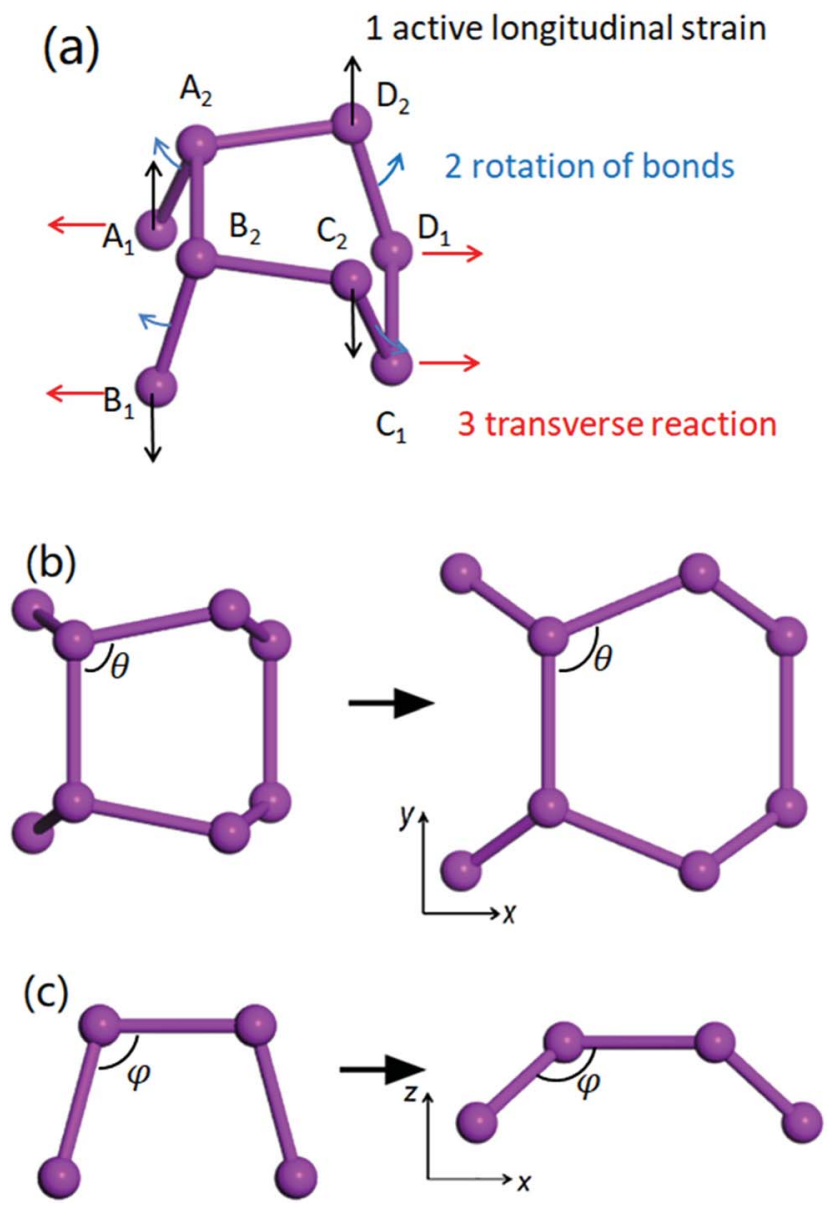

Fig. 4 (a) Deformation mechanism of the life-boat structure. A longitudinal tensile strain is applied in the $y$-direction. The black arrows represent the atoms movements in the longitudinal direction. The blue arrows show the rotation directions of the bonds. The red arrows show the final displacement of atoms in the transverse direction. The two sub-diagrams show the top view (b) and the side view (c) of the system before (left to the arrow) and after (right to the arrow) the strain.

with increasing applied tensile strain. Indeed, the thickness of both $\alpha$-phosphorene and penta-graphene obviously decrease under tensile strains. ${ }^{17,23}$ Hypothetically, if we apply an unrealistically large strain in either the $x$ - or $y$-directions, but keep the neighbouring atom is bonded at in a reasonable distance (same value as the strain-free bond distance), the atoms tend to arrange in a single atomic plane. Thus, to perform NPR, the 2D structure must be arranged in at least 2 atomic layers.

Another important feature of 2D auxetic materials is that the atoms are connected by the chemical bonds inside the unit cell or on the surface instead of on the edge, which is often described as a puckered, buckled or corrugated structure. The tetragonal or hexagonal systems under strain remain in similar shapes, but the bond angles change. SnSe, $\alpha$-phosphorene and life-boat structures have only 4 atoms at the vertices of the tetragonal unit cell, while bonds of TMDCs lie on the lateral surface of the hexagonal unit cell. The non-zero thickness and the lack of constraint on the edge are two prerequisites for an NPR in terms of the geometrical structure. 
The work done by strain can be restored both in stretched chemical bonds and distorted charge distribution caused by VSEPR. The whole system tends to distort to an away-fromequilibrium-state with lower total energy. For a given geometrical structure, rigid bonds tend to force other bonds to rotate, while soft bonds tend to be stretched. Either PPR or NPR is determined by whether the enlargement or rotation of the chemical bonds plays a dominant role in energy restoration. Both rigidity and ease of rotation are important in the auxetic phenomenon. This can be explained by the gradient of the total energy in a spherical coordinate system referred by bonds:

$$
\nabla U=\frac{\partial U}{\partial r}+\frac{1}{r} \frac{\partial U}{\partial \theta}+\frac{1}{r \sin \theta} \frac{\partial U}{\partial \varphi}
$$

where the $U$ is the total energy, $r$ is the length of the bond, $\theta$ and $\varphi$ are coordinates defined in Fig. 4. For any moment in the process under strain, the crystal tends to behave in the direction that has the minimum value - enlargement for $\frac{\partial U}{\partial r}$, rotation in a plane for $\frac{1}{r} \frac{\partial U}{\partial \theta}$ or thinning for $\frac{1}{r \sin \theta} \frac{\partial U}{\partial \varphi}$. The ratio of $\frac{\partial U}{\partial r}$ to $\frac{1}{r} \frac{\partial U}{\partial \theta}$ in $\delta$-phosphorene, $\delta$-arsenic and $\delta$-graphene under strain in the armchair direction are approximately $5: 1,2.2: 1$, and $1.2: 1$, respectively (more details see ESI $\dagger$ ).

For those NPR structures having similar charge distribution, such as life-boat phosphorene and arsenic systems, larger cohesive energy and Young's modulus imply stronger bonding. The stronger bonds force other bonds to rotate under a tensile strain, thus larger Young's modulus shows lower PR value. However, in some cases, the stronger VSEPR from neighboured bonds or lone pairs weakens this effect, often along with different charge distribution and density of states. For life-boat graphane, the VSEPR between the $\mathrm{C}-\mathrm{H}$ bond and the $\mathrm{C}-\mathrm{C}$ bond constrains the rotation of freedom and the PR of $\delta$-graphane is greater than $\delta$-phosphorene though the $\mathrm{C}-\mathrm{C}$ bond is stronger than the P-P bond. Similarly, weak $\mathrm{t}_{2 \mathrm{~g}}-\mathrm{p}$ coupling in $\mathrm{d}^{0}, \mathrm{~d}^{5}$ and $\mathrm{d}^{6}$ TMDCs causes strong repulsion among the $\mathrm{M}-\mathrm{X}$ bonds, reducing the NPR and some even become PPR. ${ }^{26}$ The mechanical formation or deformation pattern for bonds is fundamentally determined by the elemental electronic configuration, in which to achieve NPR behaviour, the bonds have to be both rigid and free for rotation.

This conclusion is not contradictory to Lakes' theory. The geometrical structure decides the mechanical reaction upon a tensile strain and depends on the electronic configuration of the elements selected, which also decides the bonding information. Fig. 5 simplifies the critical factors in the origin of NPR, which provides a route to predict 2D NPR material. In practice, starting with a discovered NPR material, first one needs to find several isostructures with different elements, then to investigate the charge density and charge distribution to compare the VSEPR. If the new structure has a similar VSEPR, the structure with greater rigidity will lead to a lower NPR. If there exists a new factor affecting the rotational freedom, then the final criterion is the comparison between the energy restored in bonds stretch and rotation under tensile strain. If the bonds

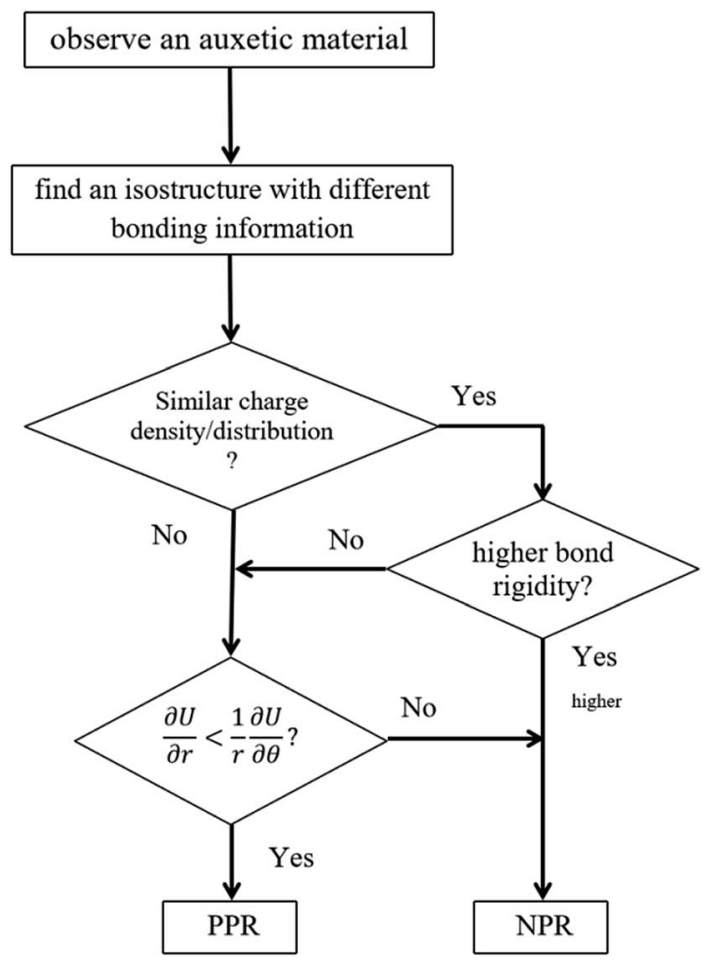

Fig. 5 The flow chart to predict new 2D NPR materials. The squares symbols are processes while the rhombus symbols are decisions.

tend to flatten then it should have NPR. To verify such an idea we have calculated the PR values of another allotrope of phosphorene $-\varepsilon$-phosphorene, ${ }^{19}$ which has a similar side view and bond information to that of $\delta$-phosphorene. Indeed, $\varepsilon$-phosphorene is found to have an isotropic NPR with the value of -0.09 . The increased PR value compared to that of $\delta$-phosphorene is due to the limitation of the rotational freedom caused by VSEPR (derived via charge distribution). For a new material with a particular structure, our proposed pattern can be utilized to estimate the likelihood of whether it has an NPR value. Yet, it remains our future endeavour to use this method to design a group of materials with controllable NPR values.

The auxetic behaviour we discuss here is in the elastic region, with the original definition of NPR as $\nu_{j i}=-\varepsilon_{j} / \varepsilon_{i}$. There is another PR defined by the formula $\nu_{j i}=-\partial \varepsilon_{j} / \partial \varepsilon_{i}$ often used under large strains. ${ }^{32-34}$ We also notice that the PR $\nu_{z y}=-\varepsilon_{z} / \varepsilon_{y}(z$ means the vertical direction and the $y$ means the armchair direction) in the $\alpha$-arsenic has been found to be negative. ${ }^{35}$ However, the positive in-plane PR reflects the increasing trend of NPR as the As-As bond is softer than the P-P bond in $\alpha$ phosphorene, which is in agreement with our conclusion. The original in-plane $\left(\nu_{x y}\right.$ or $\left.\nu_{y x}\right)$ NPR describes the intrinsic positive feedback in mechanical respond under strain.

\section{Conclusion}

Among the materials, we have selected and those mentioned in references, $\delta$-phosphorene has shown the highest PR value due to the unique concave structure and moderate VSEPR and bond 
rigidity. Based on our calculation of these isostructures and analysis of mechanical deformation, we have concluded that the NPR only reflects a positive feedback in the geometry parameter rather than in energy storage. The origin of the auxetic phenomenon can be explained as that the energy is more easily stored in the extension in the transverse direction rather than compress when a tensile strain is applied in the longitudinal direction. The auxetic behaviours are determined by not only the concave geometry structure but also the high bond rigidity and flexibility for bond rotation. The latter two factors are strongly correlated with the chemical properties of elements within the system, such as cohesive energy, VSEPR, charge distribution etc. By summarising the logical connections the NPR, we have provided an algorithmic method to discover new possible candidate 2D material through analysing the chemical structures of the isostructures of the known auxetic material.

\section{Conflicts of interest}

The authors declare no competing financial or non-financial interests.

\section{Acknowledgements}

The authors would like to thank Zijun Zhao, Jason Cheng, Hansheng Chen, and Ping Wu for the useful discussion. This research was undertaken with the assistance of resources from the National Computational Infrastructure (NCI), which is supported by the Australian Government. This work was partially supported by the Australian Research Council (DP150100018). Ruhao Fang designed the project, performed the calculations and wrote the manuscript. X. Cui and R. Zheng contributed to technical support, analysing and writing the manuscript. C. Stampfl and S. P. Ringer contributed to analysing and revising the manuscript.

\section{Notes and references}

1 R. Lakes, Adv. Mater., 1993, 5, 293-296.

2 W. Shi, W. Yang, Z.-m. Li, B.-h. Xie and M.-b. Yang, Polym. Bull., 2003, 6, 48-57.

3 F. Scarpa, IEEE Signal Process Mag., 2008, 25, 126-128.

4 J. Choi and R. Lakes, Cell. Polym., 1991, 10, 205-212.

5 Y. Sun and N. Pugno, Materials, 2013, 6, 699-712.

6 Y. J. Park and J. K. Kim, Adv. Mater. Sci. Eng., 2013, 2013, 853289.

7 J. Choi and R. Lakes, Int. J. Fract., 1996, 80, 73-83.

8 J. Choi and R. Lakes, J. Mater. Sci., 1992, 27, 5375-5381.
9 R. Lakes, Science, 1987, 235, 1038-1040.

10 G. N. Greaves, A. Greer, R. Lakes and T. Rouxel, Nat. Mater., 2011, 10, 823.

11 A. Yeganeh-Haeri, D. J. Weidner and J. B. Parise, Science, 1992, 257, 650-652.

12 D. Gunton and G. Saunders, J. Mater. Sci., 1972, 7, 10611068.

13 F. Milstein and K. Huang, Phys. Rev. B: Condens. Matter Mater. Phys., 1979, 19, 2030.

14 L. J. Gibson, Proc. R. Soc. London, Ser. A, 1982, 382, 43-59.

15 G. W. Milton, J. Mech. Phys. Solids, 1992, 40, 1105-1137.

16 N. Mounet, M. Gibertini, P. Schwaller, D. Campi, A. Merkys, A. Marrazzo, T. Sohier, I. E. Castelli, A. Cepellotti and G. Pizzi, Nat. Nanotechnol., 2018, 13, 246.

17 J.-W. Jiang and H. S. Park, Nat. Commun., 2014, 5, 4727.

18 J. Guan, Z. Zhu and D. Tománek, Phys. Rev. Lett., 2014, 113, 046804.

19 M. Wu, H. Fu, L. Zhou, K. Yao and X. C. Zeng, Nano Lett., 2015, 15, 3557-3562.

20 S. Mardanya, V. K. Thakur, S. Bhowmick and A. Agarwal, Phys. Rev. B, 2016, 94, 035423.

21 C. He, C. Zhang, L. Sun, N. Jiao, K. Zhang and J. Zhong, Phys. Status Solidi RRL, 2012, 6, 427-429.

22 L.-C. Zhang, G. Qin, W.-Z. Fang, H.-J. Cui, Q.-R. Zheng, Q.-B. Yan and G. Su, Sci. Rep., 2016, 6, 19830.

23 Z. Shunhong, Z. Jian, W. Qian, C. Xiaoshuang, K. Yoshiyuki and J. Puru, RENSIT, 2015, 7, 192-207.

24 D. Wu, S. Wang, S. Zhang, J. Yuan, B. Yang and H. Chen, Phys. Chem. Chem. Phys., 2018, 20, 18924-18930.

25 H. Wang, X. Li, P. Li and J. Yang, Nanoscale, 2017, 9, 850-855.

26 L. Yu, Q. Yan and A. Ruzsinszky, Nat. Commun., 2017, 8, 15224.

27 K. Burke, J. Perdew and M. Ernzerhof, Phys. Rev. Lett., 1997, 78, 1396.

28 S. Grimme, J. Comput. Chem., 2006, 27, 1787-1799.

29 L. Yu, A. Ruzsinszky and J. P. Perdew, Nano Lett., 2016, 16, 2444-2449.

30 B. Fulcher, X. Cui, B. Delley and C. Stampfl, Phys. Rev. B: Condens. Matter Mater. Phys., 2012, 85, 184106.

31 R. Gillespie, Coord. Chem. Rev., 2008, 252, 1315-1327.

32 D. T. Ho, S.-D. Park, S.-Y. Kwon, K. Park and S. Y. Kim, Nat. Commun., 2014, 5, 3255.

33 J.-W. Jiang, T. Chang, X. Guo and H. S. Park, Nano Lett., 2016, 16, 5286-5290.

34 R. Qin, J. Zheng and W. Zhu, Nanoscale, 2017, 9, 128-133.

35 J. Han, J. Xie, Z. Zhang, D. Yang, M. Si and D. Xue, Appl. Phys. Express, 2015, 8, 041801. 